\title{
Ouvidoria de saúde em ação: uma experiência inovadora sobre as práticas dos serviços do SUS
}

Ombudsman health in action: an innovative experience on the practices of the SUS services

\section{Ana Piterman}

Odontóloga, doutoranda em Saneamento, Meio Ambiente e Recursos Hídricos. Ouvidora de Saúde do Estado de Minas Gerais. Belo Horizonte, Brasil.

\section{Francisco Antônio Tavares Junior}

Gestor Público, mestrando em Saúde Pública. Secretário Adjunto da Secretaria Estadual de Minas Gerais. Belo Horizonte, Brasil.

\section{Fernando Antônio Leles}

Gestor Público, consultor da Organização Pan Americana de Saúde (OPAS/OMS). Brasília, Brasil.

Resumo: Investigação sobre os problemas apontados pelas queixas dos cidadãos em relação à demora e o tempo de espera na fila em receber os medicamentos na unidade de dispensação de medicamentos de alto custo da região metropolitana da Belo Horizonte. A Ouvidoria de Saúde realizou uma ouvidoria ativa na instituição para realizar uma escuta apurada sobre as principais dificuldades daquela unidade que impactavam na demora da distribuição dos medicamentos. Este estabelecimento é referência para a população de 39 municípios adstritos à SRS de Belo Horizonte em um total de 5.204.125 habitantes. A metodologia utilizada foi um estudo de campo do tipo survey exploratória descritiva. O instrumento de coleta de dados utilizado foi um questionário estruturado sobre diversos aspectos do serviço prestado para os usuários com o objetivo de recolher uma amostra do grau de satisfação/insatisfação dos usuários. Os resultados encontrados foram referentes às reclamações e solicitações dos usuários foram determinantes para que a Ouvidoria de Saúde pudesse exercer a escuta, intervir e modificar as práticas do serviço da Unidade de Dispensação e reafirmar a importância do exercício da cidadania pela sociedade, alçando-a na qualidade de agente transformador. Foram sugeridas algumas alternativas para o aperfeiçoamento dos serviços e atualmente a instituição passa por uma grande reforma na estrutura, fluxo e gestão. A ouvidoria de saúde pode atuar como instrumento de mediação entre as expectativas dos cidadãos e os serviços ofertados pelo SUS gerando melhor qualidade nas ações e aperfeiçoamento dos mecanismos de participação e acesso amplo à informação. 
Palavras-chave: ouvidoria de saúde, participação, democracia, saúde.

Keywords: health ombudsman, participation, democracy, health.

\section{Introdução}

O Componente Especializado de Assistência Farmacêutica foi aprovado pela Portaria GM/MS no. 2.981/2009 considerando os princípios e eixos estratégicos definidos pela Política Nacional de Assistência Farmacêutica, a necessidade de aprimorar os instrumentos e estratégias que asseguram e amplia - acesso da população aos serviços de saúde, incluído o acesso aos medicamentos em estreita relação com os princípios da Constituição e da organização do Sistema Único de Saúde (SUS) e ainda considerando a pactuação na reunião da Comissão Intergestores Tripartite de 24 de setembro de 2009 (Ministério da Saúde, 2010).

A definição e a regulamentação do Componente Especializado da Assistência Farmacêutica são de responsabilidade do Ministério da Saúde (MS). Assim, o elenco de medicamentos é formulado pela esfera federal podendo, a partir desta, ser modificado por cada Estado, segundo sua complexidade assistencial. As esferas estaduais são encarregadas também de formular e implementar um sistema logístico, envolvendo as etapas de seleção, programação, aquisição, armazenamento, distribuição, dispensação e promoção do uso racional dos medicamentos de dispensação excepcional.

O Art. $8^{\circ}$ da referida Portaria define o Componente Especializado como uma estratégia de acesso aos medicamentos no SUS, caracterizado pela busca da garantia da integralidade do tratamento medicamentoso, em nível ambulatorial, cujas linhas de cuidado estão definidas em Protocolos Clínicos e Diretrizes Terapêuticas (PDCT), publicados pelo Ministério da Saúde. O acesso aos medicamentos que fazem parte das linhas de cuidado para as doenças contempladas no âmbito desse componente será garantido mediante a pactuação entre a União, estados, Distrito Federal e municípios, conforme as diferentes responsabilidades definidas nesta Portaria.

A elaboração dessa lista também é importante para a melhoria da qualidade da assistência farmacêutica, uma vez que a incorporação de medicamentos tem buscado seguir, 0 que tem se preconizado 
internacionalmente, a Medicina Baseada em Evidências (MBE). Sua existência facilita a gestão da assistência farmacêutica, a uniformização de procedimentos terapêuticos e de monitoramento do tratamento, com minimização dos riscos à saúde dos pacientes e, consequentemente, melhoria das condições de saúde. Uma parte dos medicamentos deste componente são também chamados "de alto custo" e são, geralmente, onerosos demais para a população arcar com o tratamento de longo prazo, seja porque seu valor unitário é alto, seja porque, apesar de valor unitário baixo, o tratamento torna-se custoso por ser prolongado.

Eles podem ser destinados ao tratamento de doenças raras ou de baixa prevalência, tais como a doença de Crohn e lúpus eritematoso sistêmico, e também para doenças de alta prevalência, como asma grave e transtorno depressivo. Nesta última circunstância, os medicamentos serão destinados aos casos, nos quais o paciente apresentou intolerância, refratariedade aos medicamentos de primeira linha do tratamento ou quando da evolução para o quadro clínico de maior gravidade.

A Secretaria Estadual de Saúde através da Superintendência de Assistência Farmacêutica/Diretoria de Medicamentos de Alto Custo tem 28 pontos de dispensação localizados nas 18 Superintendências Regionais de Saúde (SRS) e 10 Gerências Regionais de Saúde. A SRS de Belo Horizonte possui uma Unidade de Dispensação de Alto Custo que é referência para uma população de 39 municípios adstritos a ela, num total de 5.204.125 habitantes. Essa unidade é responsável por $40 \%$ da entrega de medicamentos de alto custo do Estado e recebe cerca de 30.000 pacientes por mês.

Até 2005, a Unidade de Dispensação passou por um período de desorganização dos serviços, com o atendimento aos usuários sendo realizado de forma manual, com gestão ineficiente de estoque e falta de profissionais capacitados. As condições de atendimento eram lamentáveis pois, embora a maioria dos medicamentos já fossem entregues na atual Unidade, outros eram dispensados na Policlínica Centro Sul e Posto de Assistência Médica Saudade, do município de Belo Horizonte. O tempo de espera dos usuários para a dispensação era bastante longo (horas), além do desconforto de aguardarem em pé em um corredor insalubre. 
Em outubro de 2005, a situação foi modificada com a com a melhoria das instalações e a implantação de um sistema informatizado na dispensação. Essa foi a primeira e grande única transformação ocorrida naquela Unidade.

Ao avaliar os serviços de saúde, o principal objetivo é contribuir para a elaboração de providências que possam auxiliar e subsidiar a gestão no aperfeiçoamento da qualidade de prestação de serviços públicos. As pesquisas sobre satisfação dos usuários foram desenvolvidas na década de 1990, após a inserção e consolidação do controle social no SUS, cujos conselheiros, representantes da sociedade civil, tinham como uma das principais funções o monitoramento e a supervisão nos processos de planejamento e avaliação dos serviços (Espiridião \& Trad, 2006).

A Ouvidoria de Saúde também tem em suas diretrizes, ampliar a participação dos cidadãos usuários do SUS, garantindo-se a escuta, análise e o retorno das demandas dos cidadãos; criar um canal direto de comunicação e escuta que tenha como características a independência, autonomia e ética do sistema, preservando-se o sigilo que a atividade requer e possibilitar à Ouvidoria a avaliação contínua da qualidade dos serviços prestados pelo SUS.

É importante salientar que pesquisas dessa categoria possuem uma dimensão subjetiva, isto é, o usuário traduz a sua percepção do atendimento do SUS em sua condição relativa e tendencial. Entretanto, segundo vários autores, há uma correlação objetiva entre usuários que foram mais bem atendidos e uma maior adesão e engajamento ao tratamento, além de uma facilidade de fornecer informações à equipe de saúde, maior assiduidade na utilização dos serviços de saúde ficando mais predispostos a ter uma melhor qualidade de vida (Ware et al., 1983; Weiss, 1988 apud Espiridião \& Trad, 2006).

Segundo a teoria da realização de expectativa, o usuário demonstra maior nível de satisfação quando recebe aquilo que esperava do serviço ou excede sua expectativa. No Brasil, segundo estudos, a satisfação geralmente está associada, em primeiro lugar, a aspectos humanitários da relação médico-paciente, em seguida, ao acesso aos serviços e à disponibilidade de insumos como vacinas e medicamentos (Espiridião \& Trad, 2006).

A avaliação da qualidade dos serviços admite sempre duas dimensões: o desempenho técnico - qual seja, a aplicação do conhecimento e da tecnologia 
médica a fim de maximizar os benefícios e reduzir os riscos - e o relacionamento com o paciente. Segundo os autores citados, a avaliação da qualidade do cuidado à saúde pode dar-se em seus três componentes: estrutura (recursos utilizados pelo serviço), processo (procedimentos empregados no manejo dos problemas dos pacientes) e resultado (estado de saúde do paciente ou da comunidade resultante da interação com o serviço de saúde).

Houve várias manifestações (reclamações e solicitações) de usuários que frequentam a Unidade de Dispensação na Ouvidoria de Saúde de Minas Gerais. A Ouvidoria de Saúde decidiu atuar ativamente, isto é, foi feita uma visita técnica à instituição, com realização de entrevistas estruturadas para ouvir, sob a perspectiva dos cidadãos, quais eram os possíveis gargalos do serviço e, assim, auxiliar a gestão, demonstrando, por meio de um relatório consistente, as recomendações observadas.

\section{Material e métodos}

Para a realização do trabalho, delimitou-se o campo da pesquisa na Unidade de Dispensação de Alto Custo da Região Metropolitana de Belo Horizonte em novembro de 2011 a partir de entrevistas e análise da estrutura física e modelo de gestão. Foram entrevistados com 95 usuários com questionários estruturados. A seleção dos entrevistados foi aleatória. Por fim, o projeto avaliou o grau de satisfação, conhecimento e percepção do usuário quanto aos serviços utilizados.

Para aferir a satisfação do usuário da unidade, levou-se em consideração a natureza subjetiva desta dimensão. Com o objetivo de verificar qual a percepção do usuário especificamente em relação ao atendimento e resolutividade, além da infraestrutura do local foram propostas algumas questões sobre o atendimento da equipe, demora em adquirir o medicamento e o que o usuário apontaria sobre problemas na infraestrutura que precisaria melhorar.

As pesquisas sobre satisfação podem assim dialogar, sem se confundir, com as pesquisas sobre qualidade. Não podem ser confundidas com pesquisas de qualidade, pois a avaliação feita pelos usuários não se faz a partir da perspectiva da definição pelos saberes técnicos do que se deve entender por qualidade. Contudo, a avaliação sobre a satisfação dos usuários, entendida na sua conexão com a percepção, pode ser considerada como um dos componentes da avaliação da qualidade dos serviços. 


\section{Resultados}

Em relação à pesquisa estruturada com os usuários, constatou-se que a maioria $(57,8 \%)$ era do sexo feminino. A faixa etária predominante foi a de 41 a 60 anos, representando $54,3 \%$ dos usuários. No quesito escolaridade, a maioria dos entrevistados está representada pelo nível de escolaridade fundamental, com 46,3\% (Figura 1).

Figura 1 - Nível de escolaridade (\%) dos entrevistados.

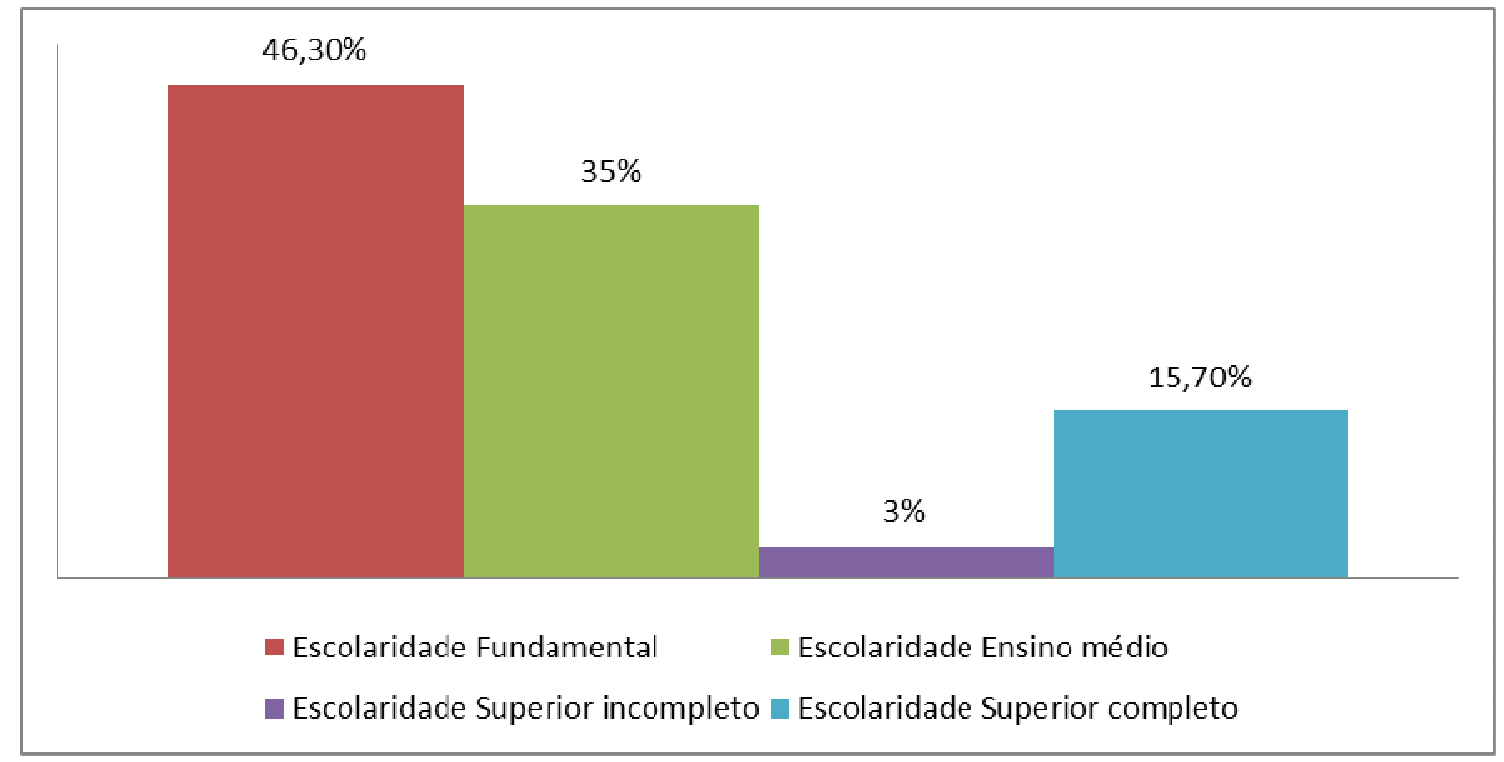

Dos entrevistados, 64,2\% nunca ouviram falar dos serviços prestados pela Ouvidoria Geral do Estado/Ouvidoria de Saúde e, embora os 22,8\% conhecessem o serviço, ainda não recorreram à mesma. Apenas $13 \%$ utilizaram o serviço da Ouvidoria de Saúde.

Com relação à qualidade de atendimento prestado pela farmácia, 72,7\% estão muitos satisfeitos (Figura 2). 
Figura 2 - Satisfação com os serviços prestados

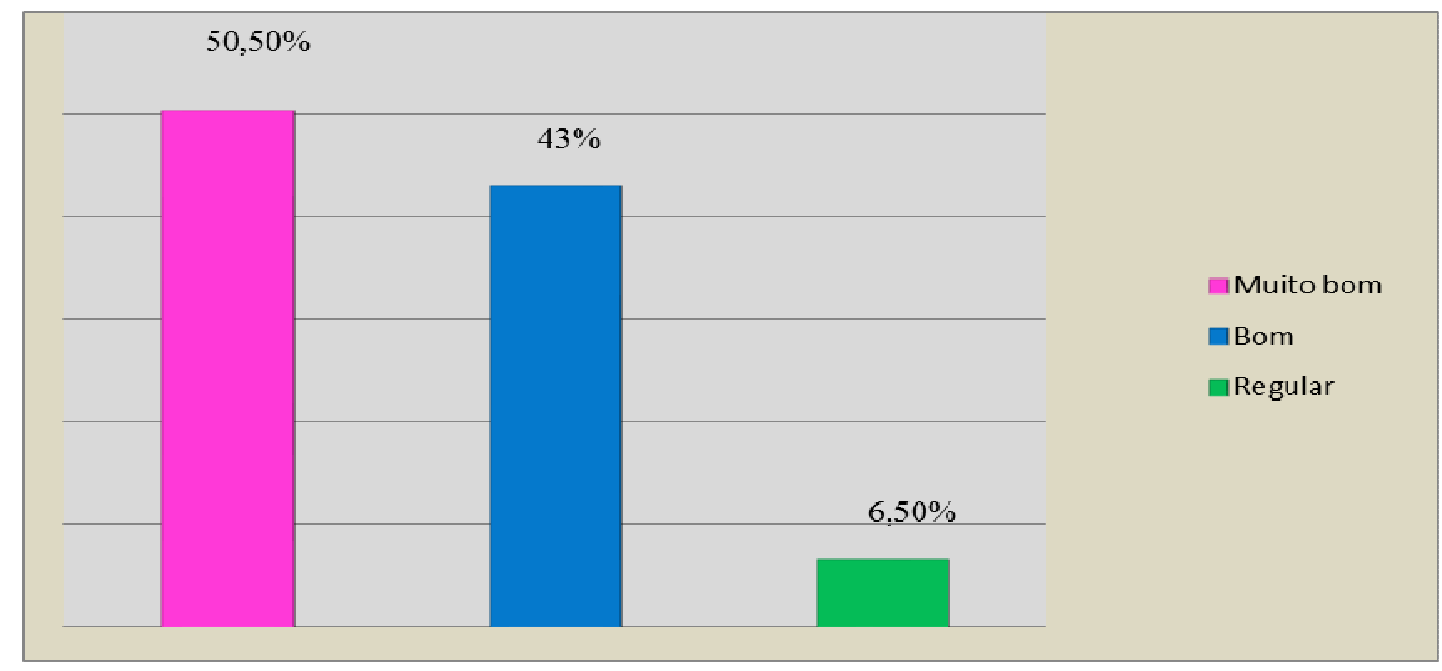

Dos entrevistados, $24,2 \%$ afirmaram que em algum momento houve falta ou atraso do medicamento (Figura 3).

Figura 3 - Supressão ou atraso na entrega dos medicamentos.

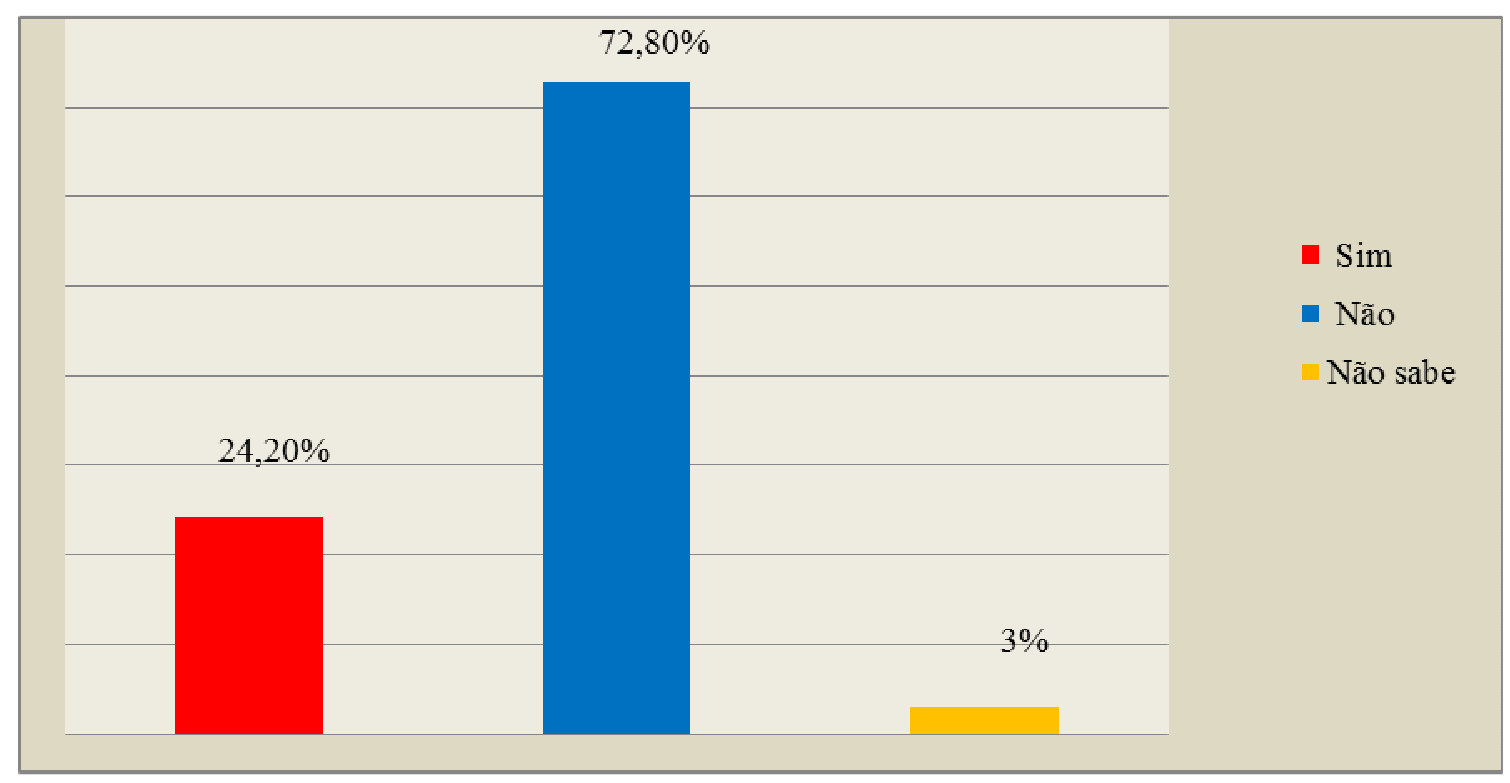

Os usuários apontaram quais os pontos que a Unidade de Dispensação deveria melhorar para prestar um serviço de qualidade (Figura 4). 
Figura 4 - Reivindicações dos usuários

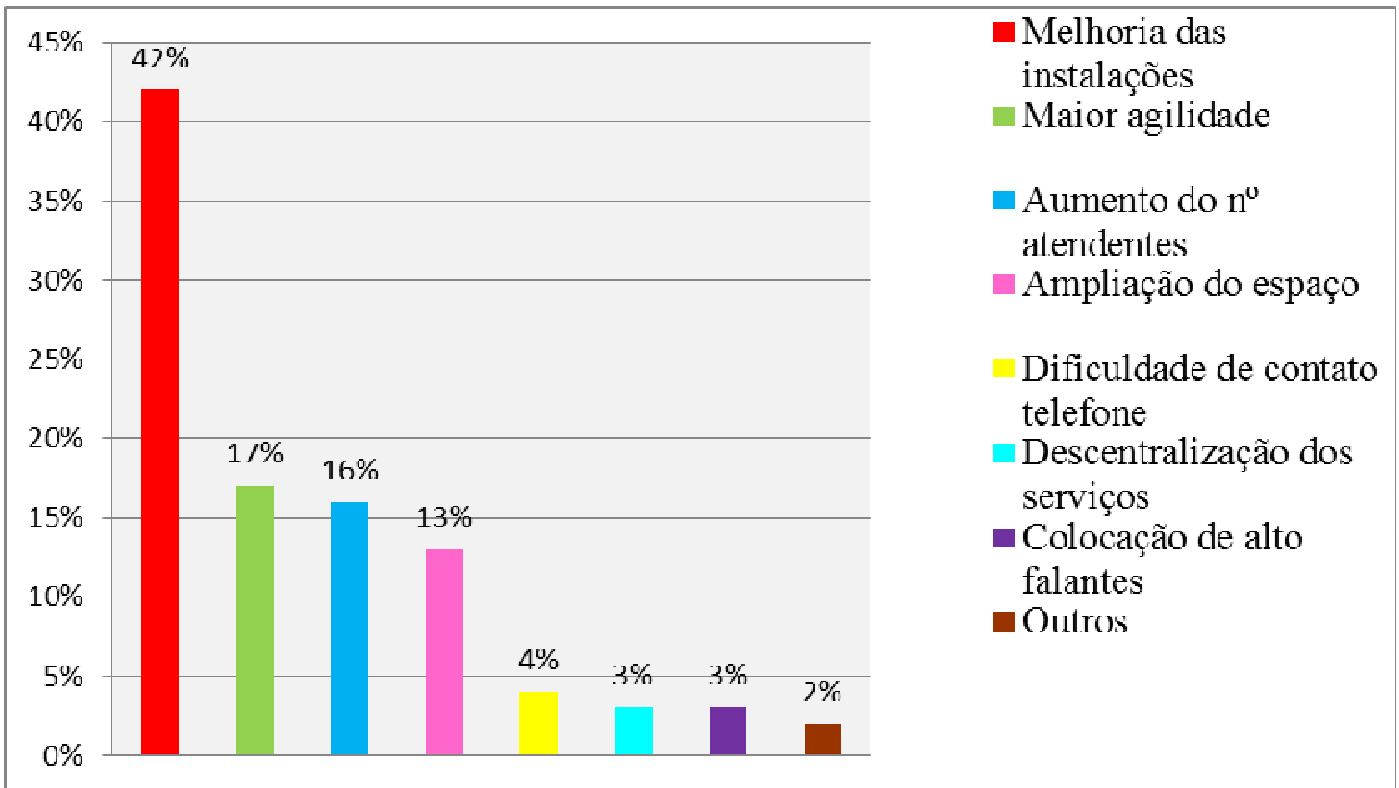

\section{Conclusões e recomendações}

Diante do exposto, para um atendimento de melhor qualidade em relação à Unidade de Dispensação de Medicamentos de Alto Custo, a Ouvidoria de Saúde do Estado de Minas Gerais recomendou que a Secretaria Estadual de Saúde se fizesse uma reforma no local, pois embora o serviço prestado fosse de excelente qualidade, o espaço físico estava subdimensionado e necessitando de reparos hidráulicos, elétricos e redistribuição de espaços prejudicando o atendimento e o trabalho dos funcionários devido à crescente demanda. Também foi recomendado que houvesse uma readequação de recursos humanos devido à crescente demanda (cerca de 2.000 usuários por dia), maior agilidade nos processos (queixa dos usuários quanto aos dias esperados pelo processo na Superintendência de Medicamentos da SES), melhor organização na distribuição de horários ao longo do dia (queixas em relação à morosidade da fila), colocação de senha eletrônica em todas as etapas para a aquisição do medicamento. Houve uma sensibilidade por parte do gestor estadual e no momento a unidade passa por reformas estruturais, além de ser lançado o programa de entrega de medicamentos em casa. 


\section{Referências}

BRASIL. Ministério da Saúde. Secretaria de Ciência, Tecnologia e Insumos Estratégicos. Departamento de Assistência Farmacêutica e Insumos Estratégicos. Da excepcionalidade às linhas de cuidado: o componente especializado da assistência farmacêutica. Brasília: Ministério da Saúde, 2010. 262 p.

ESPIRIDIÃO MA, TRAD LAB. Avaliação de satisfação dos usuários: considerações teórico-conceituais. Cad. Saúde Pública, Rio de Janeiro, 22 (6): 1267-1276, jun, 2006.

WARE JE, Snyder MK, WRIGHT WR, DAVIES AR. Defining and measuring patient satisfaction with medical care. Eval Program Plann, 6:247-63, 1983.

WEISS GL. Patient satisfaction with primary medical care: evaluation of sociodemographic and predisposition al factor. Med Care, 26:383-92, 1988. 